\title{
Effect of drying methods on the powder and compaction properties of microcrystalline cellulose derived from Gossypium herbaceum
}

\author{
Nkemakolam Nwachukwu ${ }^{\oplus 1^{*}}$, Sabinus Ifeanyi Ofoefule ${ }^{2}$ \\ 1*Department of Pharmaceutics and Pharmaceutical Technology, University of Port Harcourt, \\ Port Harcourt, Rivers State, Nigeria, ${ }^{2}$ Excipient Research and Development (ID-HEM-ERD), \\ Faculty of Pharmaceutical Sciences, University of Nigeria, Nsukka, Enugu State, Nigeria.
}

\begin{abstract}
The effect of drying method, a process variable, on the powder and compaction properties of microcrystalline cellulose (MCC) obtained from the partial acid hydrolysis of bleached alpha $(\alpha)$ cellulose content of matured linters of Gossypium herbaceum $(\mathrm{GH})$ was investigated. A portion of the wet MCC obtained was fluid bed dried at $60 \pm 1{ }^{\circ} \mathrm{C}$, inlet air of $30 \mathrm{~m}^{3} \mathrm{~min}^{-1}$ for $3 \mathrm{~h}$ (coded $M C C$-Goss F). The second portion was lyophilized at $-45 \pm 2{ }^{\circ} \mathrm{C}$ for $6 \mathrm{~h}$ (coded $M C C$-GossL). The physicochemical, scanning electron micrographs, $\mathrm{X}$ ray diffraction patterns and micromeritic properties of the derived MCCs were determined using standard methods. The cohesiveness and compactibility of the powders were investigated using Kawakita model while the deformation and compressibility pattern were determined using Heckel model. Avicel ${ }^{\circledR}$ PH $102(A V-102)$ was used as comparing standard. Ash values of $<2 \%, \mathrm{pH}(6.54 \pm 0.23$ to $6.58 \pm 0.08)$, degree of polymerization, DP (231.50) was obtained. MCC-GossF had higher moisture content, swellability, better flow indices, and lesser porosity than MCC-GossL. Kawakita model demonstrated good consolidation and compactibility for both powders. Compacts of $M C C$-Goss $L$ were significantly $(\mathrm{p}<0.05)$ harder than those of $M C C$-GossF. Heckel analysis demonstrated good compressibility and deformation pattern that was comparable with $A V$-102. Compacts of $M C C$-GossL had better mechanical and tablet compression properties than $M C C$-Goss F.

Method of drying significantly $(\mathrm{p}<0.05)$ affected the powder and compaction properties of GH MCC.
\end{abstract}

Keywords: Gossypium herbaceum. Powder. Compaction. Drying methods. Microcrystalline cellulose.

\section{INTRODUCTION}

Cellulose is an organic polymer that exists in the cell wall of all green plants with the responsibility of ensuring the existence of a mechanically stable structure (Gibson, 2012). It is a natural and almost inexhaustible resource that is produced by both plants and micro-organisms and can be used in the native or derivative form (Rajkumar, Mavikandan, Saravakumar, 2016; Suryadi, Sutriyo, Sari, Rosikhoh, 2017). Its excellent mechanical properties, low density, renewable

*Correspondence: N. Nwachukwu, Department of Pharmaceutics and Pharmaceutical Technology, University of Port Harcourt. Port Harcourt Rivers Nigeria, 500004, Nigeria. Phone: +2348068573693. Fax: 8573693. E-mail: nkemakolam.nwachukwu@uniport.edu.ng and biodegradable structure has made it a material of interest to many scientists who desire to produce environmentally friendly polymer composites or green composites (Eichorn et al., 2010). Cotton linters is one of the sources that provide the purest and highest yield of cellulose (90\%) (Setu et al., 2014).The other sources include wood pulp (mostly soft wood), agricultural waste products and hosiery wastes.

Cotton is a soft, fluffy stable fiber that grows in a boll, or protective case, around the seeds of cotton plants of the genus Gossypium in the family Malvaceae. It comprises of more than fifty species widely distributed in warm temperate to tropical zones of the world (Cope, 2018; Aliyu, Kutama, 2007). Cotton most commonly refers to the linter or boll of Gossypium species and the 
commonly cultivated species amongst them include: Gossypium herbaceum Linn, Gossypium arboreum Linn, Gossypium hirsutum Linn, and Gossypium barbadensis Linn. (Okujagu, 2009). Gossypium herbaceum is a species of cotton native to the semi-arid regions of sub-Saharan Africa and Arabia where it still grows wildly as a perennial or annual shrub (Yalli et al., 2015). In Nigeria, wild and cultivated species are found in the central and northern States/parts of the country (Aliyu, Kutama, 2007).

Microcrystalline cellulose is a purified partially depolymerized nonfibrous form of cellulose obtained by treatment of $\alpha$-cellulose with mineral acids (Thoorens et al., 2014). It is available as a fine, white, odourless crystalline powder. Literature search shows that MCC is one of the pharmaceutical excipients that have been most widely accepted and used as a direct compression excipient because of its superior quality as a strong dry binder, tablet disintegrant, anti-adherent, absorbent, filler and diluent (Thoorens et al., 2014). The food and cosmetic industry have also found it useful as a suspension stabilizer and water retainer (Chuayjuljt, Su-uthai, Charuchinda, 2010; Nwachukwu, Ofoefule, 2017). The characteristics of most MCC may show a variation based on the source of pulp used as raw material and the method of processing it (Natarajan, Kumaravel, Palanivelu, 2016). Some commercial brands of MCC such as Avicel $\AA$, Emcocel $\AA$, Comprecel ${ }^{\circledR}$ and Microcel ${ }^{\circledR}$ may show variations in the degree of crysrallinity, moisture content, surface area, porous structure, and molecular weight. These physical differences possessed by each brand would affect the products functionality when used as an excipient (Azubuike, Odulaja, Okhamafe, 2012).

However, when a pulp is used as starting material, besides variations of the chemical processing steps, method of drying may cause a variation in the characteristics of the resultant MCC. Most commercial MCC are dried using the spray drying technique which influences some of its characteristics such as the porosity, structure and discreteness of the dried product. Method of drying using lyophilization involves freezing the wet substance (MCC) and causing the ice to sublime directly to vapour by exposing it to a low partial pressure of water vapour. This is expected to introduce a high inter particulate porous nature to the MCC which in turn would affect the particle characteristics such as the morphology, shape, porosity and aggregation (Nwachukwu, Ofoefule, 2017). Fluid bed drying method aids drying of the powder as discrete particles that do not have a high inter particulate void.

This work aims to investigate the effect of two drying methods, fluid bed and lyophilized drying on the powder and compaction characteristics of microcrystalline cellulose obtained from GH. Avicel ${ }^{\circledR}$ PH 102, a commercial brand of MCC prepared by spray drying, was used as comparing standard.

\section{MATERIAL AND METHODS}

\section{MATERIAL}

Matured cotton linters, sodium hydroxide (Merck, Germany), hydrochloric acid, magnesium nitrate and corn starch (BDH, Poole England), sodium hypochlorite $3.5 \% \mathrm{w} / \mathrm{v}\left(\mathrm{JIK} \AA\right.$, Reckitt and Colman Nig. Plc), Avicel ${ }^{\circledR}$ PH 102 (FMC Biopolymers, USA), potassium sulphate, sodium chloride (J.T. Baker, New Jersey, USA), talc, magnesium stearate (Sigma, USA) and distilled water (generated in Pharmaceutical Technology Laboratory, University of Port Harcourt, Nigeria).

\section{METHODS}

Matured cotton linters were purchased from local farmers at Katsina, Katsina State, Nigeria and were identified in the Department of Pharmacognosy, University of Port Harcourt, Port Harcourt, Rivers State, Nigeria as Gossypium herbaceum. The bolls which were already sun-dried at ambient conditions were manually sorted for stalk, hair leaves and petioles.

\section{Extraction of cellulose and $\alpha$ cellulose.}

The method of Ohwoavworhua and Adelakun with slight modification was adopted (Ohwoavworhua, Adelakun, 2005). A quantity of $500 \mathrm{~g}$ of the sorted and dried cotton fiber was soaked for $12 \mathrm{~h}$ in $95 \% \mathrm{v} / \mathrm{v}$ ethanol, and after squeezing off the alcohol it was digested with $8.0 \mathrm{~L}$ of a $2.5 \% \mathrm{w} / \mathrm{v}$ sodium hydroxide solution at a boiling temperature of $100{ }^{\circ} \mathrm{C}$ on a sand bath for $3 \mathrm{~h}$ in a $15 \mathrm{~L}$ stainless steel vessel with intermittent stirring during digestion. The resultant pulp was washed with cold distilled water until neutral and then strained using a muslin cloth. Initial bleaching was done with $10 \mathrm{~L}$ of $0.4 \% \mathrm{w} / \mathrm{v}$ solution of sodium hypochlorite at $80{ }^{\circ} \mathrm{C}$ for 30 min. Removal of chlorine was achieved by washing with distilled water until neutral to litmus. The material 
was strained through a muslin cloth and further digested with $7.0 \mathrm{~L}$ of $17.5 \% \mathrm{w} / \mathrm{v}$ solution of sodium hydroxide at $100{ }^{\circ} \mathrm{C}$ for $1 \mathrm{~h}$. Further washing with copious amounts of distilled water was done until neutral to litmus. After straining, the resultant material was bleached a second time with $0.4 \% \mathrm{w} / \mathrm{v}$ solution of sodium hypochlorite at $80{ }^{\circ} \mathrm{C}$ for $30 \mathrm{~min}$ to obtain $\alpha$ cellulose. It was washed till neutral with distilled water, strained through a fine muslin cloth and dried in batches in a stainless steel tray placed in a hot air oven (Mermmert ${ }^{\circledR}$, Germany) at $60{ }^{\circ} \mathrm{C}$ for $2 \mathrm{~h}$. The $\alpha$ cellulose obtained was weighed and stored. The procedure was repeated to obtain more $\alpha$ cellulose.

\section{Production of microcrystalline cellulose}

A quantity of $50 \mathrm{~g}$ of the $\alpha$ cellulose obtained from $\mathrm{GH}$ was weighed into a $2 \mathrm{~L}$ glass beaker and treated with $1.0 \mathrm{~L}$ of $2.5 \mathrm{~N}$ Hydrochloric acid solution at a temperature of $105 \pm 2{ }^{\circ} \mathrm{C}$ with constant vigorous stirring using a glass rod for $15 \mathrm{~min}$ on a paraffin oil bath. The hot acid mixture was poured into cold water, allowed to cool down and washed severally with cold distilled water until neutral to litmus. The resultant MCC was strained using a muslin cloth. More MCC was obtained by repetition of this process. The damp MCC was divided into two portions for drying. A portion was dried in a fluid bed dryer (Sherwood ${ }^{\circledR}$ Tonado model, China) at a temperature of $60 \pm 1{ }^{\circ} \mathrm{C}$ and inlet air of $30 \mathrm{~m}^{3} \mathrm{~min}^{-1}$ for $3 \mathrm{~h}$ and was coded MCC-GossF. The second portion was lyophilized using a model LGT 18 freeze dryer (Gallenkamp $\AA$, England) set at a temperature of $-45{ }^{\circ} \mathrm{C}$ for $6 \mathrm{~h}$ and was coded MCCGossL. Milling and sizing of the resultant MCC dried by either method was achieved using a domestic electric blender (Binatone ${ }^{\circledR}$, Japan) and passed through a 250 $\mu \mathrm{m}$ stainless steel sieve (Retsch ${ }^{\circledR}$, Germany). It was weighed and stored for further experiments.

\section{RAW MATERIAL CHARACTERIZATION}

\section{Physicochemical evaluation}

\section{Identification}

The colour, odour, taste and texture of the MCC were observed and recorded. Test for presence of starch was done by soaking a $2 \mathrm{~g}$ portion of the MCC in iodine solution for $5 \mathrm{~min}$ after which the excess iodine was drained off. Visual observations were made for any changes in colour and the results were recorded. Two drops of $60 \% \mathrm{v} / \mathrm{v}$ of sulphuric acid solution were added to a fresh portion of the MCC and observation for any colour change to the MCC was also made and recorded (BP, 2012). These tests were done for all the MCCs.

\section{Solubility}

Distilled water was added drop wise to a 1.0 $\mathrm{g}$ portion of the MCC until it was submerged. It was vigourously shaken, visually observed for solubility and observations recorded. The procedure was repeated using acetone, $0.1 \mathrm{~N}$ hydrochloric acid $(\mathrm{HCl})$ solution and ethanol. These tests were done with each MCC powder and the results recorded.

\section{pH determination}

A quantity of $2 \mathrm{~g}$ of each sample of MCC was dispersed in $100 \mathrm{~mL}$ of distilled water with vigorous shaking for $5 \mathrm{~min}$. The dispersions were allowed to settle and the $\mathrm{pH}$ of the supernatants determined (BP, 2012) using a $\mathrm{pH}$ meter $(\mathrm{PHS} \AA 25$, India). Three replicate determinations were made and the results recorded.

\section{Total ash content}

A quantity of $3 \mathrm{~g}$ each of MCC-GossF, MCCGossL and AV-102 were independently combusted in a furnace at $550{ }^{\circ} \mathrm{C}$ for $5 \mathrm{~h}$. The total ash content was determined by the measurement of the residue left after the combustion.

\section{Elemental/Heavy metal analysis}

The microcrystalline cellulose obtained from $\mathrm{GH}$ was analyzed for the presence of heavy metals such as Lead $(\mathrm{Pb})$, Iron $(\mathrm{Fe})$, Zinc $(\mathrm{Zn})$, Manganese $(\mathrm{Mn})$ and Arsenium (As). Digestion of $1.0 \mathrm{~g}$ of the MCC using $10 \mathrm{~mL}$ of a medium made up of concentrated Nitric acid ( $\mathrm{HNO} 3)$ and $\mathrm{HCl}$ in a ratio of $1: 3$ was done. It was filtered and the filtrate made up to $50 \mathrm{~mL}$ using distilled water. Pure/standard samples of the heavy metals being investigated were diluted serially to enable determination of the maximum wavelengths of absorption and standard calibration plot (Beers plot) of the elements. An Atomic absorption spectroscope, AAS (Model AA-7000, ROM version 1.01, S/N 
A30664700709 SHIMADZU®, Japan) was used to determine the quantities of $\mathrm{Pb}, \mathrm{Fe}, \mathrm{Zn}, \mathrm{Mn}$ and $\mathrm{As}$ present at wavelengths of 283.33, 248.30, 213.90, 279.50 and $193.7 \mathrm{~nm}$, respectively. All the determinations were done at a slit setting of $0.2 \mathrm{~nm}$ of the spectroscope and at a lamp current of between $10-12$ milliAmpere (mA). The absorbance without a sample in the atomizer was measured and this was repeated with a sample in the atomizer. The ratio between the two absorbance values obtained was converted to the sample concentration or mass using the Beers plot earlier determined.

\section{$X$-ray Diffraction $(X R D)$}

A quantity of $150 \mathrm{mg}$ of MCC-GossF, MCCGossL and AV-102 were independently tightly packed in the sample holder and exposed to the X-ray radiation using an X-ray diffractometer machine (D/MAX-1200, Rigaku ${ }^{\circledR}$, Japan) powered by a 45 kilovolt (kv) X-ray generator (RINT 2000, Rigaku ${ }^{\circledR}$, Japan) at an input of $35(\mathrm{~mA})$ and set at $0.020^{\circ}$ and step time of $29.10 \mathrm{sec}$ $\left(\mathrm{Cu} \mathrm{K} \alpha\right.$ radiation) and a scan speed of $2^{\circ}$ per sec at a 2 theta $(\theta)$ range of $3-80^{\circ}$. Diffractograms of each MCC was recorded and the degree of crystallinity of each sample calculated from Equation 1 (Gurdag et al., 2001).

Crystallinity index $(\mathrm{C} . \mathrm{I})=\left[\left(\mathrm{I}_{002}-\mathrm{I}_{\mathrm{am}} / \mathrm{I}_{\mathrm{am}}\right)\right]$ x 100 Eq.1

Where $I_{002}$ is the highest peak intensity of the crystalline fraction and Iam is the low intensity peak of the amorphous region.

\section{Scanning Electron Microscopy (SEM) test}

A Scanning Electron Microscope (Phenom Prox ${ }^{\circledR}$, Model MVE016477830, Eindhoven Netherland) was used to determine the morphology, particle shape and size. Quantities ranging from 3-5 $\mathrm{mg}$ of MCCGossF, MCC-GossL and AV-102 were placed in appropriate containers in the sample chamber and covered with a coating/sputter of gold (Q15ORES, Quorum, England). The equipment was switched on and readings were taken.

\section{Swelling capacity determination}

The method of Bowen and Vadino (1984) with slight modification was used to determine the swelling capacity of the microcrystalline cellulose samples MCC-GossF, MCC-GossL and AV-102. A $3 \mathrm{~g}$ quantity of the sample was placed in a graduated glass measuring cylinder and tapped to obtain the tapped volume, Vt. A dispersion of the powdered sample was made in $85 \mathrm{~mL}$ of distilled water and was agitated thoroughly. The volume was made up to 100 $\mathrm{mL}$ with more distilled water and it was allowed to stand undisturbed for $24 \mathrm{~h}$ on a flat surface and the volume of the sediment formed, Vv noted. Triplicate determinations were done for each of the samples and the swelling capacity calculated as a percentage using Equation 2 (Bowen, Vadino, 1984).

Swelling capacity $($ S.C. $)=\left[\left(\mathrm{V}_{\mathrm{v}}-\mathrm{V}_{\mathrm{t}}\right) / \mathrm{V}_{\mathrm{t}}\right] \times 100 \quad$ Eq.2

\section{Hydration capacity}

The Kornblum and Stoopaks method (Kornblum, Stoopak, 1973) for the determination of hydration capacity was adopted with slight modification. A quantity of $1 \mathrm{~g}$ of each sample was weighed into a $15 \mathrm{~mL}$ plastic centrifuge tube and a $10 \mathrm{~mL}$ volume of distilled water added to each tube. This was shaken intermittently over a $2 \mathrm{~h}$ period and left to stand for $30 \mathrm{~min}$. Using a table top centrifuge model TX 150 (ThermoFisher Scientific, UK) centrifugation was done for $10 \mathrm{~min}$ at 1000 rotations per minute (rpm) and the supernatant carefully decanted. The wet sediment left was weighed and the hydration capacity calculated as:

Hydration capacity (H.C.) $=\mathrm{x} / \mathrm{y}$

Eq.3

Where $\mathrm{x}$ is the weight of the wet sample/powder sediment, and $y$ is the weight of the dry sample/powder.

\section{Moisture content}

The moisture content of MCC-GossF, MCC-GossL and AV-102 were determined by placing $5 \mathrm{~g}$ of each powder independently in a tarred white porcelain crucible kept in a hot air oven (Mermmet ${ }^{\circledR}$, England) and drying done at $105{ }^{\circ} \mathrm{C}$ until a constant weight was obtained. The percentage moisture content was determined using Equation 4 (Okhamafe, Azubuike, 1994).

Moisture content $=100 *\left[\left(\mathrm{~W}_{\mathrm{i}}-\mathrm{W}_{\mathrm{f}}\right) / \mathrm{W}_{\mathrm{i}}\right]$

Eq.4

Where Wi is the initial weight of powder before drying and Wf is the final weight of powder after drying. 


\section{Moisture sorption test}

A quantity of $2 \mathrm{~g}$ each of MCC-GossF, MCCGossL and AV-102 were placed on the surface of a $7 \mathrm{~mm}$ tarred Petri dish and placed in desiccators of relative humidities of $52,75,84$ and $96 \%$ respectively at ambient temperature. The weight gained over a five (5) day period was calculated for each sample from Equation 5. Triplicate determinations were made.

Moisture sorbed $=\left[\left(\mathrm{W}_{2}-\mathrm{W}_{1}\right) / \mathrm{W}_{1}\right] \times 100$

Where $\mathrm{W}_{1}$ is the weight before exposure and $\mathrm{W} 2$ is the weight after exposure

\section{Particle size analysis}

The methodology employed was that of the nest of sieves. A cascade of 7 stainless steel sieves (Retsch ${ }^{\circledR}$, Germany) ranging from $1 \mathrm{~mm}$ to $45 \mu \mathrm{m}$ arranged in descending order with a collection pan underneath the sieves was placed on a sieves shaker (Retsch ${ }^{\circledR}$ Ltd, Germany). The sieves were weighed empty and $25 \mathrm{~g}$ sample of each of MCC-GossF, or MCC-GossL or AV102 was placed on the top most sieve and agitated for 5 min at an amplitude of $1.5 \mathrm{~mm} / \mathrm{g}$ and 57 stops. At the end of the test, the weight of material retained on each of the sieves was obtained by deducting the weight of the empty sieves from its weight with the powder sample retained. Triplicate determinations were done and the percentage of MCC retained was calculated using Equation 6.

Percentage mass $=[$ mass powder retained $/$ total mass powder] $x 100$

Eq.6

The fineness of the MCCs was classified by determining the smallest sieve opening through which a known quantity of the MCC powder could pass through. The smallest sieve opening through which 10, 50 and $90 \%$ or more of the particles passed through were denoted as the $\mathrm{D}_{10}, \mathrm{D}_{50}$ and $\mathrm{D}_{90}$ respectively (USP, 2009). The cumulative percentage of the MCC retained on each sieve was calculated against the sieve size where it was retained and the mean cumulative percentage of powder retained on each sieve was plotted against the sieve size in a graph and extrapolations of the particle sizes made at 10,50 and $90 \%$ for MCC-GossF, MCC-GossL and AV-102.

\section{Degree of polymerization and molecular weight}

Solution viscometry method was used in the determination of the degree of polymerization and molecular weight of the MCCs (Sihtola et al., 1963) A U-tube viscometer (Technicu size C100) and an ammoniacal solution of copper was the apparatus and solvent used. A stock solution of $2 \% \mathrm{w} / \mathrm{v}$ of MCC-GossF, MCC-GossL and AV-102 were separately made. Serial dilutions to obtain concentrations of $1 \%, 0.5 \%, 0.25 \%$ $0.125 \%$ were made from each of the stock solutions. Measurements were made of the flow time for each MCC solution at the different dilutions available and the flow rates determined. The dilutions were considered adequate when the flow time measurements obtained using the viscometer for each of the serially diluted MCC solutions were close or equal to that of the solvent (ammoniacal solution of copper). The experiments were conducted at ambient temperature. Density determinations of the different MCC solutions were carried out using a pycnometer. From the relative viscosity (viscosity ratio), the reduced viscosity (viscosity number) was measured. The intrinsic viscosity was determined from the plot of reduced viscosity versus concentration as the intercept of the straight line portion of the slope on the y axis (reduced viscosity) section of the plot. The degree of polymerization was calculated from Equation 7 (Sihtola et al., 1963).

$(\mathrm{DP})^{0.85}=1.1 \times \eta$

Eq.7

where $\eta$ is the intrinsic viscosity.

The molecular weight can be calculated from Equation 8

$\mathrm{DP}=\mathrm{M} / \mathrm{M}_{\mathrm{o}}$ Eq. 8

Where $\mathrm{M}$ is the molecular weight of the MCC, and Mo is the molecular weight of glucose.

Calculations and plots to determine the intrinsic viscosity, relative viscosity, the molecular weight and degree of polymerization were done for MCC-GossF, MCC-GossL and AV- 102.

\section{Micromeritic properties}

\section{Bulk and tapped density}

The bulk density was determined by pouring a 10 g quantity of each powder sample freely under gravity 
into a $50 \mathrm{~mL}$ clean, dry, graduated measuring cylinder. The volume occupied by the powder was noted as the bulk volume $(\mathrm{Vb})$. The bulk density, $\mathrm{Db}$ was calculated from Equation 9 (Staniforth, 1988):

$\mathrm{D}_{\mathrm{b}}=\mathrm{M} / \mathrm{V}_{\mathrm{b}}$

where $\mathrm{M}$ is the mass of material or powder.

The tapped volume was determined by dropping/ tapping the cylinder on a flat wooden platform from a height of about $2-3 \mathrm{~cm}$ at $2-3 \mathrm{sec}$ intervals until there was no further reduction in the volume of the material. The tapped density, Dt was calculated using Equation 10:

$\mathrm{D}_{\mathrm{t}}=\mathrm{M} / \mathrm{V}_{\mathrm{t}}$

Where $\mathrm{M}$ is mass of the powder or sample, $\mathrm{Vt}$ is tapped volume and Dt is tapped density

\section{Hausner's quotient (ratio) and Carr's index}

The Hausner's quotient and Carr's index (Carr's compressibility) for each powder sample were calculated from Equations 11 and 12 (France et al., 2017).

Hausners quotient (H.Q.) $=\mathrm{D}_{\mathrm{t}} / \mathrm{D}_{\mathrm{b}}$ Eq.11

Carr's Index $($ C.I. $)=\left[1-\left(D_{b} / D_{t}\right)\right] \times 100$

\section{Particle density}

The liquid displacement method was employed in the determination of the particle density of each of the microcrystalline cellulose powder samples. A pre-weighed $25 \mathrm{~mL}$ volume pycnometer was filled with xylene, stoppered, and excess fluid wiped off the pycnometer. This was weighed and its weight denoted as a. The pycnometer was emptied and $1 \mathrm{~g}$ of the powdered sample was weighed into it, and it was refilled with xylene, stoppered, wiped clean of excess fluid and reweighed. The particle density was calculated from Equation 13 (Achor, Oyeniyi, Yahaya, 2014):

$P_{d}=W_{p} /\left[\left(a+W_{p}\right)-b\right] \times S . G$.

Where Pd is the particle density, S.G. is specific gravity of the solvent, a is the weight of pycnometer and solvent, Wp is weight of powder and b is weight of pycnometer + solvent + powder. Triplicate determinations were conducted for each powder sample.

\section{Flow rate and angle of repose}

The dynamic angle of repose was measured using the fixed funnel and free standing cone method. A 10 g quantity of microcrystalline cellulose powder was poured into a clamped stoppered clean glass funnel whose orifice was $4 \mathrm{~cm}$ above a flat surface. The powder was allowed to flow freely under gravity from the funnel unto a $6.5 \mathrm{~cm}$ diameter platform. The time it took the powder to flow through the funnel, and height of the powder heap formed were measured and recorded. The flow rate and tangent of the powder heap were calculated using Equations 14 and 15 respectively:

F.R. $=$ M / F.T.

Eq.14.

Where F.R. is the flow rate, F.T. is the flow time and $M$ is the mass of powder used.

Angle of repose $(\theta)=\tan ^{-1}(\mathrm{~h} / \mathrm{r})$

Eq.15

Where $\mathrm{h}$ is the height and $\mathrm{r}$ the radius of the heap of powder.

\section{Powder porosity}

Powder porosity, $€$ is obtained when the values of the particle density, Pd and bulk density, Db are fitted into Equation 16 (Neuman, 1967).

$\epsilon=\left[1-\left(D_{b} / P_{d}\right)\right] \times 100$

Eq. 16

\section{Compactibility and powder cohesion}

The cohesion and compactibility of the microcrystalline powders were derived from the Kawakita plots by determining the tapped density and powder behaviour as the powder was being tapped. The Kawakita Equation describes what happens in a confinement of a powder column between the volume reduction of powder in the column and the applied pressure. A $10 \mathrm{~g}$ quantity of the microcrystalline powder was poured freely into a $50 \mathrm{~mL}$ clean, dry, graduated, glass measuring cylinder and the bulk volume, Vo noted. The mechanical tapping of the cylinder at determined 
incremental number of taps, $\mathrm{N}$ led to a systematic reduction in the volume occupied by the powder after each tapping until there was no further decrease or reduction in volume, $\mathrm{V}$. The degree of volume reduction, $\mathrm{C}$ was calculated from the values of $\mathrm{V}$ and Vo. The degree of cohesion and compactibility of the powder was calculated from the Kawakita Equation, Equation 17 (Frenning, Mahmoodi, Nordstrom, 2009).

$N / C=N / a+1 / a b$

Eq. 17

Where $\mathrm{C}$ is derived from $(\mathrm{Vo}-\mathrm{V}) / \mathrm{Vo}$. The cohesiveness of the powder sample is given as $1 / \mathrm{b}$ while the compactibility of the powder is given as a. A plot of $\mathrm{N} / \mathrm{C}$ against $\mathrm{N}$ has its slope as $1 / \mathrm{a}$ while $1 / \mathrm{ab}$ is the intercept. The procedure was carried out on MCCGossF, MCC-GossL and AV-102.

\section{Compaction of microcrystalline powders}

The microcrystalline powders: MCC-GossF, MCC-GossL and AV-102 were each compressed at different compression loads ranging from 4.90 to 14.71 MPa (MegaPascal). A flat faced set of punches with a diameter of $10 \mathrm{~mm}$ was used to make 100 compacts of $300 \mathrm{mg}$ weight per compact for each batch. Each powder was manually fed into a single punch hydraulic tablet press (Model C, Carver Inc., Winscosin, USA) and compressed for $30 \mathrm{sec}$. Lubrication of the punches and dies was done with a dispersion of stearic acid powder in acetone before each compaction cycle. Compacts were evaluated $24 \mathrm{~h}$ post compression.

\section{Evaluation of microcrystalline cellulose compacts}

A $24 \mathrm{~h}$ post compression-relaxation time was allowed before evaluation for weight uniformity, thickness, hardness, friability, disintegration time and tensile strengths of the compacts.

\section{Weight uniformity test}

Twenty compacts randomly selected from each batch of MCC-GossF, MCC-GossL and AV-102 were individually weighed. Calculations were done for the mean, standard deviation and coefficient of variance. The criterion for acceptance or rejection of the compacts was based on the British Pharmacopoeia tolerance limits for uncoated tablets of $250 \mathrm{mg}$ and above (BP, 2012).

\section{Thickness/height and diameter test}

Ten compacts randomly selected from each of the batches of the compacts were measured with the aid of a micrometer screw gauge for thickness and diameter. The mean and standard deviation of results for thickness was calculated while the tablet diameter was also recorded for each batch.

\section{Hardness test}

The hardness of ten compacts randomly selected from each batch of MCC-GossF, MCC-GossL and AV-102 were determined using a TBH 200 model hardness tester (Erweka ${ }^{\circledR}$, Germany). The mean, standard deviation, and coefficient of variance were determined.

\section{Disintegration test}

One compact out of six compacts randomly selected from each batch of MCC was put into the cylindrical hole of each of the six glass tubes of the basket assembly of a double basket disintegration tester (model ZT-3, Erweka ${ }^{\circledR}$, Germany) and each compact held in place with a glass disc. Each beaker was filled with $500 \mathrm{~mL}$ of $0.1 \mathrm{~N} \mathrm{HCl}$ and heated up to $37 \pm 1{ }^{\circ} \mathrm{C}$. The time it took each compact to break up and completely pass through the mesh was noted. Triplicate determinations were made.

\section{Friability test.}

Ten tablets randomly selected from each batch of the compacts were freed of dust by directing a stream of air at them. They were collectively weighed and put in one of the drums of a twin drum electronic friabilator (Model TAR 200, Erweka ${ }^{\circledR}$, Germany) programmed to revolve at 25 revolutions per minute (rpm) for $4 \mathrm{~min}$. The compacts were collected and de-dusted and any broken tablets rejected. The tablets were reweighed and the abrasion resistance $(\mathrm{F})$ calculated from Equation 18 (Armstrong, 1990):

$\mathrm{F}=100\left[\left(\mathrm{~W}_{\mathrm{o}}-\mathrm{W}\right) / \mathrm{W}_{\mathrm{o}}\right.$ Eq.18

Where Wo is the initial weight and $\mathrm{W}$ is the final weight. 
Tensile strength

The determination of the tensile strength of the compacts was based on the hardness, thickness and diameter. Calculations were done using Equation 19:

Tensile strength $\left(\mathrm{T}_{\mathrm{S}}\right)=2 \mathrm{P} / \varpi \mathrm{dt}$

Where $\mathrm{P}$ is the breaking force or hardness, $\mathrm{d}$ is the tablet diameter, $\mathrm{t}$ is the tablet thickness.

\section{Heckel analysis}

Heckel analysis involves the application of the Heckel Equation in relating the relative density of a powder bed to the applied compression load during tableting. It describes the densification behavior of the powder bed from the point of die filling to the time a measured pressure/load is applied. It also describes the deformation mechanism of the powder in the formation of the compact. The Equation is stated as shown in Equation 20 (Heckel, 1963):

$\ln (1 / 1-\mathrm{D})=\mathrm{KP}+\mathrm{A}$

where $\mathrm{D}$ is the relative density of a powder compact at pressure $\mathrm{P}, \mathrm{K}$ is a constant to measure the plasticity of the compressed material, A is the Y-axis intercept, related to die filling and particle rearrangement before deformation and bonding of the separate particles. The yield pressure, Py is obtained as the reciprocal of the slope of the straight line portion of the plot, K. From the value of $\mathrm{A}$, the relative Density, $\mathrm{D}_{\mathrm{A}}$, can be calculated using Equation 21:

$\mathrm{D}_{\mathrm{A}}=1-\mathrm{e}^{-\mathrm{A}}$

The relative density of the powder bed at the point the applied pressure is zero is denoted as Do. It represents the initial re-arrangement phase of densification as a result of die filling and is derived as the ratio of the loose density to the particle density. The relative density, $D_{B}$, describes the re-arrangement of particles at the initial stages of compression and is dependent on the theoretical point of densification at which deformation of the particles commences. It is derived from Equation 22:

$\mathrm{D}_{\mathrm{B}}=\mathrm{D}_{\mathrm{A}}-\mathrm{D}_{\mathrm{O}}$

\section{Re-working potential of microcrystalline cellulose}

Batches of compacts of the MCCs compressed at the different compression loads were triturated to fine particles using a Wedge wood mortar, screened through a $250 \mu \mathrm{m}$ stainless sieve and recompressed at the same compression loads and target compact weights of 300 $\mathrm{mg}$ each using $10 \mathrm{~mm}$ flat faced punches and the same hydraulic tablet press (Model C, Carver Inc., Winscosin, USA). Reworked compacts were evaluated $24 \mathrm{~h}$ postcompression for uniformity of weight, thickness, hardness and friability as described earlier. Plots of hardness against compression pressure were made for the initial compacts and the reworked compacts of each sample. The area under the curve (AUC) of each plot was determined using Wolfram/Alpha Widget ${ }^{\circledR}$ software. The re-working potential was calculated as the percentage of AUC of re-worked MCC (AUCr) against AUC of initial MCC (AUCi) compact. This is shown in Equation 23.

Re-working potential $=\left(\mathrm{AUC}_{\mathrm{r}} / \mathrm{AUC}_{\mathrm{i}}\right) \times 100$ Eq. 23

\section{Statistical evaluation}

The data obtained were statistically analyzed using ANOVA and students t test (SPSS version 21). Values were considered significant at $\mathrm{p}<0.05$.

\section{RESULTS AND DISCUSSION}

\section{Physicochemical properties}

The yield of $\alpha$ cellulose and microcrystalline cellulose were $81.38 \%$ and $65.55 \%$ respectively with reference to the dry GH linters. The MCCs obtained were white, tasteless, odourless and fine powder which resembled AV-102 in these parameters reported. Some other test results such as that for identification using iodine and sulphuric acid (Table I) show the absence of starch and confirm that the sample is MCC (BP, 2012). Solubility profile results (Table I) show that the materials were insoluble in water, acetone, alcohol and dilute mineral acid (BP 2012). This can be attributed to their high crystalline content and structure (Achor, Oyeniyi, Yahaya, 2014; Gwon et al., 2010). However, they were completely dissolved in ammoniacal solution of copper tetramine. The $\mathrm{pH}$ was between $6.54 \pm 0.23$ to $6.75 \pm 0.71$ indicating the neutrality of the samples which qualifies 
them for use in the formulation of acidic and basic drugs (BP, 2012). A DP of 231.50 and 231.62 for MCC-GossL and MCC-GossF respectively was comparable to the value of 234.40 obtained for AV-102 (Table I). Generally, MCC should have DP lower than 350 (BP 2012; Carlin et al., 2007). Percent crystallinity index values obtained ranged from 81.25 to $83.33 \%$ which is consistent with MCC (Rowe, Mckilop, Bray, 1994). Figure 1 shows the X-ray diffractograms of MCC-GossF, MCC-GossL and AV-102. The similarity in diffraction pattern was evident amongst them and the peaks seen attests to the existence of crystal lattice with high degree of orderliness which is associated with MCC. However, the double peaks seen in the diffractograms of MCC-GossF and MCC-GossL at $20.5^{\circ}$ of $2 \theta$ may be attributed to the presence of cellulose II crystalline structure while the single peak of AV-102 is indicative of cellulose I (Kolpak, Blackwell, 1976; Kuga, Brown, 1988; Gwon et al., 2010). The similarity (double peaks) found in both MCC-GossF and MCC-GossL is as a result of the application of the same chemical $(\mathrm{NaOH})$ treatment to both during processing while that of the commercial brand, AV-102 may have been as a result of it being processed by a different chemical method. The SEM micrographs of MCC-GossL and AV-102 were similar (Figure 2) and the particles were seen existing as aggregates or clusters while MCC-GossF was seen as single strands/particles (Figure 2). This implies that the drying methods affected the morphologies, however, the images are consistent with SEM micrographs for MCC that has been reported by other reseachers (Natarajan, Kumaravel, Palanivelu, 2016; Rajkumar, Mavikandan, Saravanakumar, 2016). SEM micrographs enable the quantitative extraction of information of the surface texture of a material (Gomez et al., 2009). The ash content values of the MCCs from GH were significantly $(\mathrm{p}<0.05)$ lower than that of AV-102. This shows that organic residues were minimal in the GH MCCs and this can be attributed to the raw material source and method of preparation employed. Hydration capacity of AV-102 was slightly higher than that of MCC-GossF and MCC-GossL (Table I). This can be attributed to the lower crystallinity index of AV-102 (Gwon et al., 2010). The moisture content/loss on drying results showed that MCC-GossF retained more moisture than MCC-GossL and AV-102 (Table 1). However, the percentage moisture retained by the MCCs was within BP acceptable limits of 5 to $7 \%$ when dried at oven temperature of $105{ }^{\circ} \mathrm{C}$ (BP 2012). Moisture content has been reported to affect the compaction properties, tensile strength, and viscoelastic properties of MCC (Amidon, Houghton, 1995; Sun, 2008). The moisture hysteresis evaluation results showed that all the MCCs adsorbed moisture at the different relative humidities they were exposed to or subjected. The percentage moisture adsorbed increased as the relative humidity condition increased and MCCGossF adsorbed more moisture than AV-102 and MCCGossL. The total amount of water adsorbed in a material has been reported to be proportional to the fraction of amorphous material contained (Amidon, Houghton, 1995). The quantity of moisture adsorbed could affect the stability of the formulation containing such MCC especially when used with drugs that can easily undergo hydrolysis in the presence of moisture (Tomar, Singh, Sinha, 2016). However, the swelling capacity of MCCGossL was higher than that obtained for MCC-GossF and AV-102. The hydration capacity indicates the amount of water a material is able to absorb on hydration while swellablity indicates increase in volume of water taken up after absorption (Thoorens et al., 2014). These values obtained (Table I) show that tablets formulated with this excipient would likely disintegrate readily and the possible mechanism would be by wicking and swelling and subsequent destruction of the existing hydrogen bonds within the tablets (Fox, 1963; Thoorens et al., 2014). Elemental analysis results show the MCCs to be safe for heavy metals. They were completely absent for lead $(\mathrm{Pb})$ and arsenic (As) while others such as iron, manganese, zinc and sodium were present within World Health Organization (WHO) tolerance limits (Table I). (Onyem, Onyem,Usese, 2015). The particle sizes at $\mathrm{D}_{10}$, for MCC-GossF, MCC-GossL and AV-102 were 74.00 $\pm 1.20,74.50 \pm 1.85$ and $75.50 \pm 1.10 \mu \mathrm{m}$ respectively; the $\mathrm{D}_{50}$ for MCC-GossF, MCC-GossL and AV-102 were $158.00 \pm 1.60,155.00 \pm 1.55$ and $150.00 \pm 1.05 \mu \mathrm{m}$ respectively; and $\mathrm{D}_{90}$ for MCC-GossF, MCC-GossL and AV-102 were $240.00 \pm 0.95,235.00 \pm 1.00,234.00 \pm$ $1.80 \mu \mathrm{m}$ respectively. The United States Pharmacopoeia, 2009 classifies powders whose particles range from 90 to $125 \mu \mathrm{m}$ as very fine, 125 to $180 \mu \mathrm{m}$ as fine and 180 to $325 \mu \mathrm{m}$ as moderately fine (USP, 2009). Using $\mathrm{D}_{50}$ as the measuring parameter, the powders can be classified as fine. 
TABLE I - Some physicochemical properties of MCC-GossF, MCC-GossL and AV-102

Sample/
Parameter $\quad M C C-G o s s F \quad M C C-G o s s L$

Iodine solution

Sulphuric $\operatorname{acid}(60 \% \mathrm{v} / \mathrm{v})$

Solubility in water, alcohol, acetone, dilute mineral acid.

Solubility in ammoniacal solution of copper tetramine.

$\mathrm{pH}$

Ash content (\%)

$\%$ crystallinity

Molecular weight

Degree of polymerization

Hydration capacity
Reddish brown colour

Blue colour

Insoluble

Completely soluble

$6.54 \pm 0.23$

0.28

83.33

$41,706.09$

231.50

$2.55 \pm 0.04$

$8.70 \pm 0.92$

$3.40 \pm 0.55$

$2.30 \pm 1.02$

$1.50 \pm 1.00$

0.00

0.00

0.12

0.12

2.87

0.00

$118.01 \pm 2.60$

$7.18 \pm 1.00$
Reddish brown colour

Blue colour

Insoluble

Completely soluble

$6.56 \pm 0.08$

0.28

81.94

$41,727.71$

231.62

$2.86 \pm 0.07$

$6.70 \pm 0.35$

$2.30 \pm 0.22$

$0.80 \pm 0.94$

$0.30 \pm 0.48$

0.00

0.00

0.12

0.12

2.87

0.00

$97.20 \pm 4.76$

$6.78 \pm 1.20$
Reddish brown colour

Blue colour (ppm)

Loss on drying (\%)

NMT is not more than. 


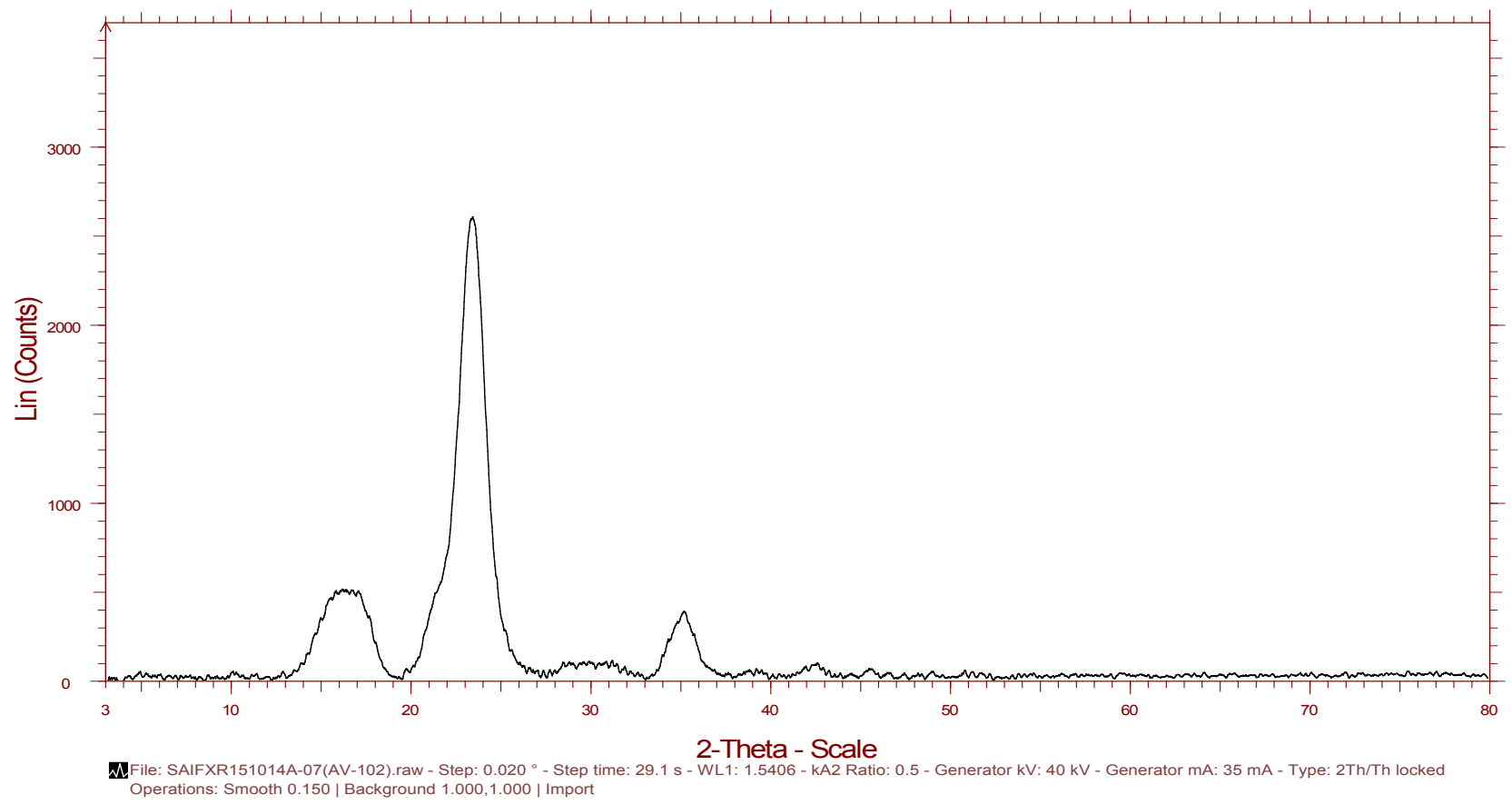

FIGURE 1 - X-ray diffractograms of AV-102, MCC-GossL and MCC-GossF.

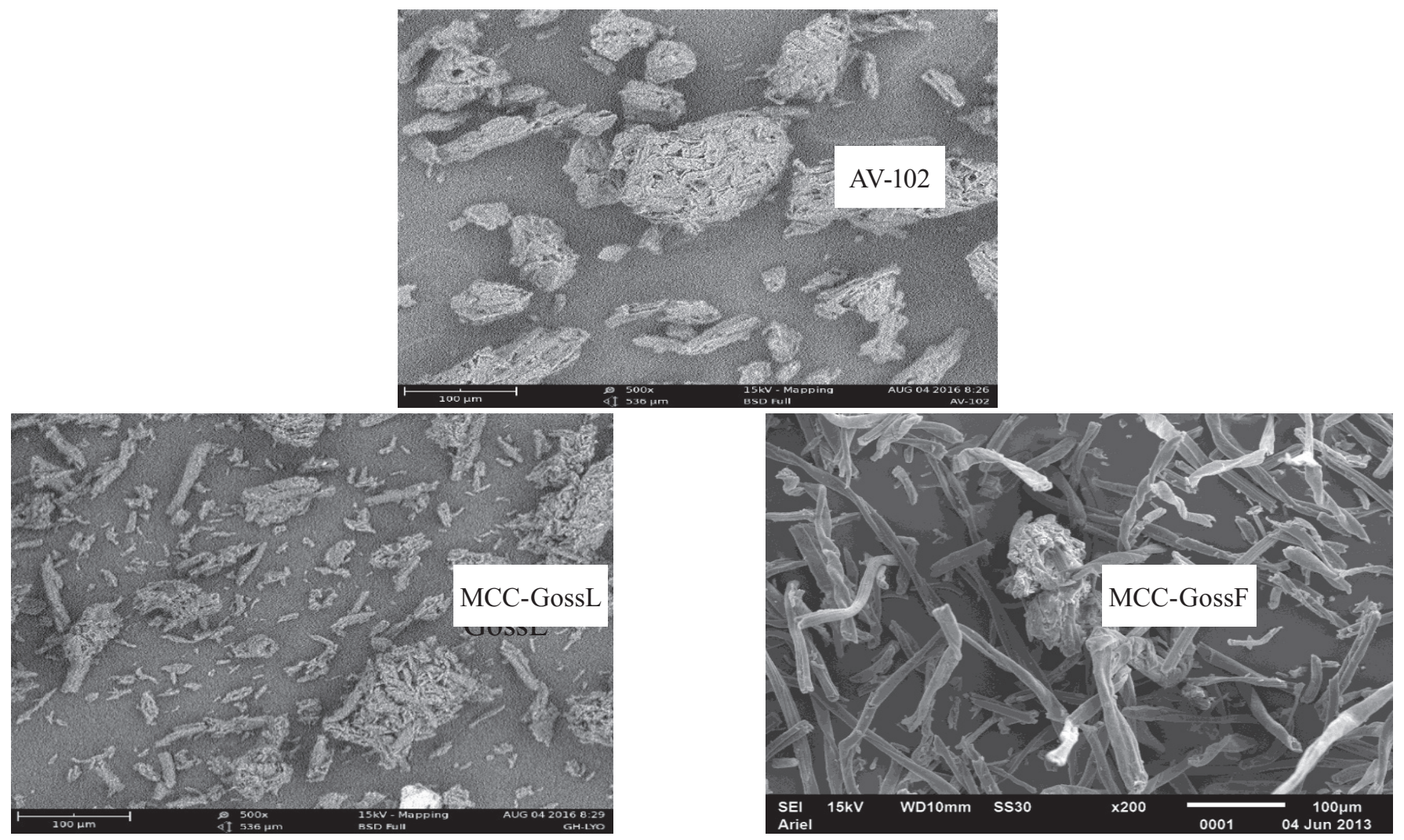

FIGURE 2 - Scanning Electron Micrographs of AV-102, MCC-GossL and MCC-GossF. 


\section{Micromeritic properties}

Some of the flow properties of the powders are shown in Table II. The bulk and tapped density values indicate that the powders would densify on tapping or agitation of the powder bed. However, the density values of MCC-GossF were higher than those of MCC-GossL. The packing fraction of MCC-GossF was higher than that of AV-102 while MCC-GossL had the lower value (Table II). The porosity values of the MCCs were high and this infers a high percentage of void spaces within each powder. The porosity of MCC-GossL was the highest while that of MCC-GossF was the least. These high porosity characteristic would impair the free flow of these materials (Azubuike, Odulaja, Okhamafe, 2012). Considering the angle of repose (Table II) the powders were classified as excellent. However, considering the Hausner's quotient and Carr's index classification, they were classified as good, fair and passable for MCCGossF, AV-102 and MCC-GossL respectively (USP 2009, BP 2009). Thus the flowability of MCC-GossF and AV-
102 were better than MCC-GossL. The Kawakita plots gave linear graphs (Figure 3). The compactibility, a, and cohesiveness, $1 / \mathrm{b}$, derived from these are shown in Table III indicating good compactibility and consolidation behavior for all the MCCs. Compactibilty depicts the level of volume reduction of a powder as pressure is put on the powder as a result of tapping and the lower the value the more compactible the powder is considered (Alderbborn, Nystron, 1982; Autamashih et al., 2011). The compactibility value of 0.22 obtained for both AV-102 and MCC-GossL is higher than that for MCCGossF (0.15). It has been shown from experimental correlations that the higher the compactibility, the lesser the cohesiveness (Yamashiro, Yausa, Kawakita, 1983). The cohesiveness or fluidity of a powder is its resistance to flow which is as a result of attractive forces on the powder particles (Turki, Fatah, 2008). The cohesiveness of MCC-GossF (7.33) was lower than those of AV-102 and MCC-GossL whose values were 9.09 respectively. Thus the propensity to flow of MCC-GossF was higher than that of AV-102 and MCC-GossL.

TABLE II - Some micromeritic properties of MCC-GossF, MCC-GossL and AV-102

\begin{tabular}{|c|c|c|c|c|}
\hline \multicolumn{2}{|l|}{$\begin{array}{l}\text { Sample/ } \\
\text { Parameter }\end{array}$} & $M C C$-GossF & MCC-GossL & $A V-102$ \\
\hline \multicolumn{2}{|c|}{ Bulk density (g/mL) } & $0.53 \pm 0.00$ & $0.37 \pm 0.01$ & $0.31 \pm 0.04$ \\
\hline \multicolumn{2}{|c|}{ Tap density (g/mL) } & $0.60 \pm 0.03$ & $0.47 \pm 0.01$ & $0.38 \pm 0.02$ \\
\hline \multicolumn{2}{|c|}{ Angle of repose $\left({ }^{\circ}\right)$} & $26.59 \pm 2.65$ & $36.28 \pm 1.76$ & $30.52 \pm 2.35$ \\
\hline \multicolumn{2}{|c|}{ Flow rate $(\mathrm{g} / \mathrm{s})$} & $5.46 \pm 3.56$ & Poor flow & $5.23 \pm 0.22$ \\
\hline \multicolumn{2}{|c|}{ Carr's index (\%) } & $12.42 \pm 0.47$ & $21.81 \pm 1.45$ & $18.96 \pm 0.67$ \\
\hline \multicolumn{2}{|c|}{ Packing Fraction } & $0.88 \pm 0.01$ & $0.78 \pm 0.02$ & $0.81 \pm 0.01$ \\
\hline \multicolumn{2}{|c|}{ Hausner's quotient } & $1.14 \pm 0.02$ & $1.27 \pm 0.04$ & $1.23 \pm 0.01$ \\
\hline \multicolumn{2}{|l|}{ Porosity (\%) } & $63.64 \pm 0.00$ & $80.10 \pm 0.27$ & $76.12 \pm 0.36$ \\
\hline \multicolumn{2}{|c|}{ Particle density $(\mathrm{g} / \mathrm{mL})$} & $1.42 \pm 0.17$ & $1.54 \pm 0.03$ & $1.56 \pm 0.07$ \\
\hline \multirow{3}{*}{$\begin{array}{l}\text { Particle size } \\
(\mu \mathrm{m})\end{array}$} & $\mathrm{D}_{10}$ & $74.00 \pm 1.20$ & $74.50 \pm 1.85$ & $75.50 \pm 1.50$ \\
\hline & $\mathrm{D}_{50}$ & $158.00 \pm 1.60$ & $155.00 \pm 1.55$ & $150.00 \pm 1.05$ \\
\hline & $\mathrm{D}_{90}$ & $240.00 \pm 0.95$ & $235.00 \pm 1.00$ & $234.00 \pm 1.80$ \\
\hline
\end{tabular}




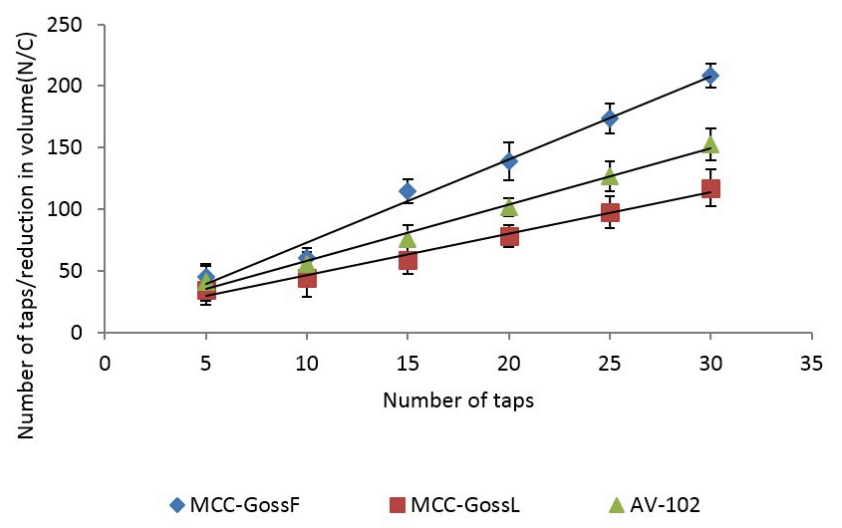

FIGURE 3 - Kawakita plots of MCC-GossF, MCC-GossL and AV-102.

TABLE III - Compactibility and cohesiveness of microcrystalline cellulose powders

\begin{tabular}{|c|c|c|c|c|c|c|}
\hline Sample & a & b & $1 / a$ & $1 / b\left(P_{k}\right)$ & 1/ab (\%) & $\mathbf{R}^{2}$ \\
\hline$M C C-G o s s F$ & 0.15 & 0.08 & 4.56 & 7.33 & 5.60 & 0.992 \\
\hline MCC-GossL & 0.22 & 0.11 & 6.74 & 9.09 & 12.42 & 0.989 \\
\hline AV-102 & 0.22 & 0.11 & 3.39 & 9.09 & 12.42 & 0.992 \\
\hline
\end{tabular}

\section{In vitro Compact properties}

Compact weights ranged from $296.05 \pm 1.79$ to $301.60 \pm 2.48 \mathrm{mg}$ for all the samples analyzed. These values fall within the British Pharmacopoeia acceptable range of $\pm 5 \%$ variation for uncoated tablets weighing above $250 \mathrm{mg}$ (BP, 2012). The thickness of the compacts reduced as compression pressure increased. Figure 4 shows a plot of the hardness of the MCC compacts as a function of compression pressure. Generally, there was an increase in hardness of the compact as compression load was increased from 4.7 to $14.3 \mathrm{MPa}$. The hardness of compacts was in the range of AV-102 > MCC-GossL > MCC-GossF. This reflects the strengths of the compacts and this is also expected to affect the strengths of the tablets that would be formulated with these excipients. The disintegration time of the compacts is shown in Figure 5. All compacts disintegrated within $15 \mathrm{~min}$ and therefore conform to British Pharmacopoeia specification of not more than 15 min for uncoated tablets (BP, 2012). This can be attributed to the high intra-particle porosity possessed by MCC which in turn promotes water uptake into the matrix core by capillary action and disruption of the hydrogen bonds (Bolhuis, Chowhan, 1996). MCC is estimated to have an internal surface area of approximately 90 to $95 \%$ of each particles entire surface area (Rowe, Mckillop, Bray, 1994). Disintegration times of MCC-GossF compacts were lower than MCC-GossL and AV-102 compacts. Similarly, the compact porosity values ranged from $3.61-31.37 \%, 2.87-21.24 \%$ and $2.87-17.02 \%$ for MCC-GossF, MCC-GossL and AV-102 respectively. The porosity of the compacts decreased as the compression pressure increased. The porosity of a tablet is one of the factors that determine the hardness and the tensile strength of the tablet. It also affects the disintegration and dissolution behavior of the compact. The higher the porosity, the more easily water or other aqueous media would penetrate into the tablet 
thereby enhancing disintegration and subsequently dissolution of the API it contains (Wu et al., 2007). The friability of the compacts were between $0.20 \pm 0.04$ to $0.76 \pm 0.01 \%, 0.30 \pm 0.02$ to $0.82 \pm 0.01 \%, 0.20 \pm 0.04$ and $0.85 \pm 0.01 \%$ for MCC-GossL, MCC-GossF and AV-102 respectively. Generally, MCC-GossL was less friable than MCC-GossF compacts and the friability decreased as the compression pressure increased. This is attributable to higher particulate bonds that exists within the plastically deformed particles of each compact (Adolfsson, Gustasson, Nystrom, 1999). All the compacts met with friability specification for uncoated tablets which is given as $\leq 1 \%$ (BP 2012; USP, 2009). The compacts are therefore expected to be able to withstand undesirable abrasion and shocks that may be encountered during its transportation and handling.

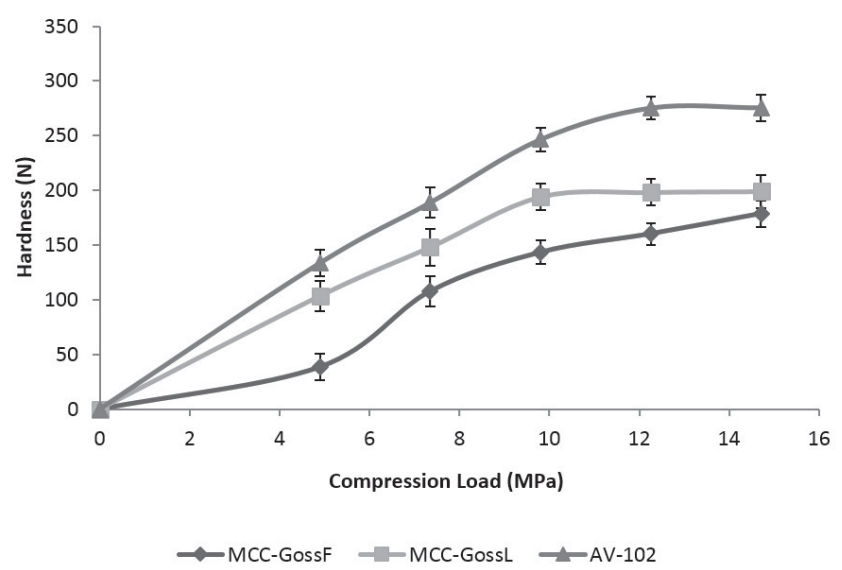

FIGURE 4 - Hardness plots of MCC-GossF, MCC-GossL and $A V-102$.

Table IV shows the Heckel values for MCCGossF, MCC-GossL and AV-102. The intercept A was derived from extrapolation of the straight line portion of the plot (Figure 6). The yield pressure, Py describes the tendency of the material to plasticize or fragment under an applied pressure which can be related to its compressibility (Heckel, 1961). MCCGossF and MCC-GossL had values of 0.79 each while AV-102 had 0.22. This indicates that both MCCGossF and MCC-GossL exhibited a faster plasticity or onset of deformation than AV-102. Do represent the initial packing in the die as a result of filling. The Do value of MCC-GossF was highest while that of AV-102 was lowest, implying that the fluid bed dried powder exhibited the highest degree of packing and re-arrangement on die filling while AV-102 exhibited the least. $\mathrm{D}_{\mathrm{A}}$ represents the total degree of packing at zero and low pressures, while $\mathrm{D}_{B}$ represents the particle rearrangement phase in the early compression stage and shows the extent of particle fragmentation (Automashih et al., 2011).

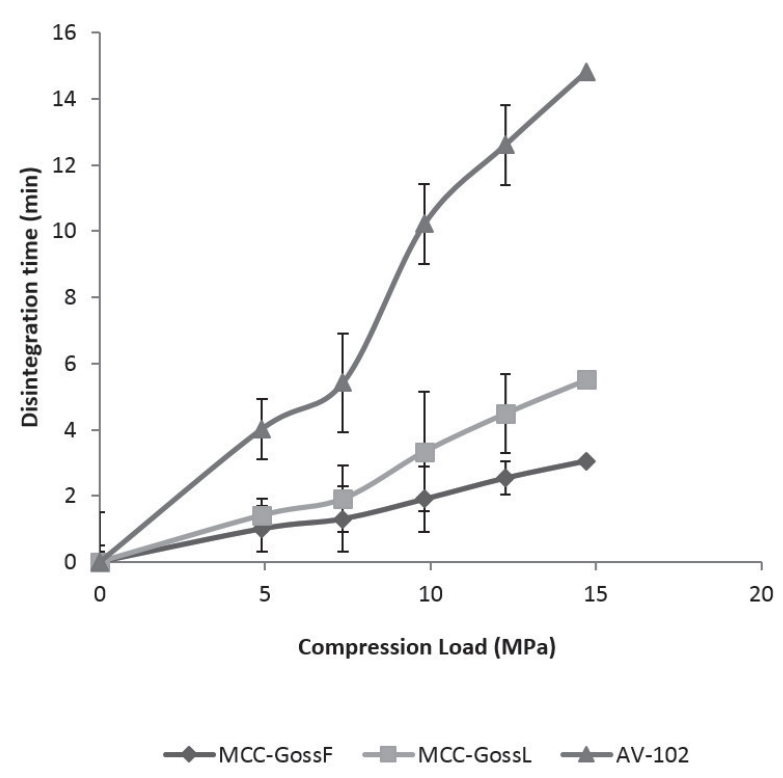

FIGURE 5 - Disintegration plots of MCC-GossF, MCC-GossL and $A V-102$.

TABLE IV - Parameters of Heckel plot

\begin{tabular}{lcccccc}
\hline Sample & $\mathbf{K}$ & $\begin{array}{c}\mathbf{P y} \\
\left(\mathbf{M N m}^{-2}\right)\end{array}$ & $\mathbf{D}_{\mathbf{0}}$ & $\mathbf{D}_{\mathbf{A}}$ & $\mathbf{A}$ & $\mathbf{R}^{2}$ \\
\hline $\begin{array}{l}\text { MCC- } \\
\text { GossF }\end{array}$ & 5.59 & 0.79 & 0.37 & 0.89 & 0.15 & 1.00 \\
$\begin{array}{l}\text { MCC- } \\
\text { GossL }\end{array}$ & 5.59 & 0.79 & 0.24 & 0.42 & 0.13 & 0.95 \\
AV-102 & 4.46 & 0.22 & 0.19 & 0.91 & 0.21 & 0.98
\end{tabular}

Figure 7 is a plot showing the results of the percentage re-working potential of the powders. The results show that all the MCCs could be reworked. The lyophilized powder was the most re-workable while the fluid bed dried (MCC-GossF) was the least. This implies slight loss of some of their initial characteristics when passed through a stress situation such as compaction which could cause fragmentation and plasticity. They have 
the ability to retain their mechanical properties which would result in the production of tablets with minimal weight variation, good tensile strength, friability and disintegration when used in tablet formulations.

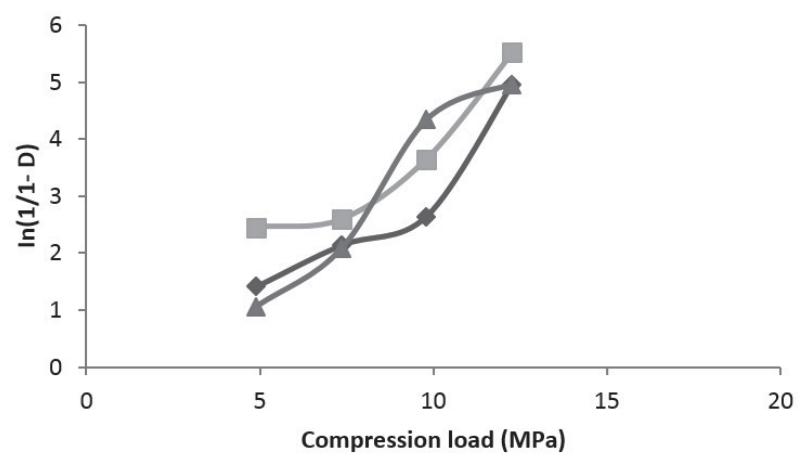

$\longrightarrow$ AV-102 $\rightarrow$ MCC-GossF $\rightarrow$ MCC-GossL

FIGURE 6 - Heckel plot of $M C C$-GossF, MCC-GossL and $A V$ 102.

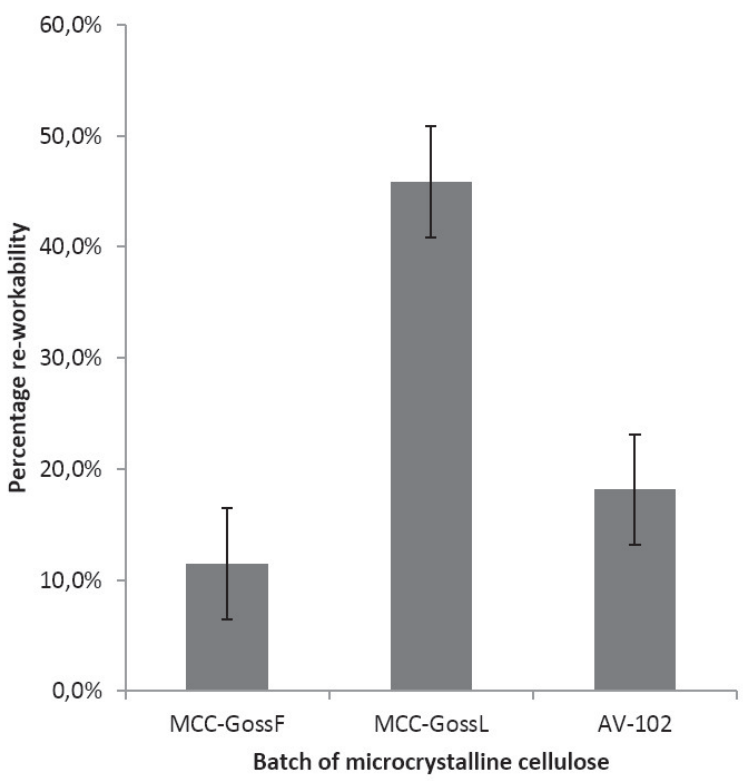

FIGURE 7 - Reworking potential of $M C C$-Goss F, MCCGoss $L$ and $A V-102$.

\section{CONCLUSION}

The yield of MCC obtained from (GH) was high as expected, since cotton linter is one of the world's highest sources of cellulose. The results of the powder characterization tests show that method of drying significantly affected the physical characteristics of the MCCs derived from Gossypium herbaceum. This is evidenced in the flow and compaction behavior of the MCCs. The micromeritic indices show that MCC-GossF had higher values in terms of bulk and tapped density, packing fraction, and mean particle size. It also has a lower Hausner's quotient, Carr's index, and porosity. These parameters were responsible for the better flow properties in comparison to the MCC-GossL. In terms of densification, volume reduction, and compactibility as assessed by the Kawakita model, MCC-GossL had higher values than MCC-GossF but was comparable to AV-102. In terms of compressibility and mechanical strength, MCC-GossL had compacts that were significantly $(\mathrm{p}<0.05)$ stronger than $\mathrm{MCC}-$ GossF. The lower bulk and tapped density values, higher porosity, higher surface area (lower mean particle diameter), and pattern of deformation could have been responsible for the superior mechanical properties obtained. Evaluation of the compacts showed the hardness, tensile strength and disintegration times of MCC-GossL compacts to be higher than that of MCC-GossF. The friability of MCC-GossL was lower than that of MCC-GossF. However, all these parameters were within the BP 2012 acceptable limits for uncoated tablets and thus the compacts can be assessed as of good quality. These parameters of MCC-GossL were comparable to AV-102. Based on the Heckel analysis, all the MCCs showed good slippage and plasticity (deformation) even at the application of low pressures. These results show that the compacts produced from MCC that was dried by lyophilization were significantly better $(\mathrm{p}<0.05)$ in terms of mechanical and compressional properties than the compacts produced from MCC that passed through the fluid bed method of drying. Based on these results, lyophilized method of drying is preferable over fluid bed drying in the production of GH-MCC.

\section{ACKNOWLEDGEMENT}

We deeply appreciate FMC Biopolymers, Brussels, Belgium sales office for their kind donation of Avicel $\mathrm{PH}$ 102. Our thanks also go to Mr. Godwin Jacob Effiong and Dr. Akpabio, E.I. of the Department of Pharmaceutics and Pharmaceutical Technology, University of Uyo, Akwa Ibom State, Nigeria for providing technical assistance in the processing of the materials.

\section{CONFLICT OF INTEREST: Nil}




\section{REFERENCES}

Achor M, Oyeniyi YJ, Yahaya A. Extraction and Characterization of microcrystalline cellulose obtained from the back of the fruit of Lageriana siceraria (water gourd), J App Pharm Sci., 2014;4(1):057-060.

Adolfsson A, Gustafsson C, Nystrom C. Use of tablet tensile strength adjusted for surface area and mean interparticulate distance to evaluate dominant bonding mechanisms, Drug Dev. Ind. Pharm., 1999:25.753-746.

Alderborn G, Nystrom. Studies on direct compression of tablets IV, the effect of particle size on the mechanical strength of tablets. Acta Pharma Suecica, 1982:19.381-390.

Aliyu BS, Kutama AS.Assessing the potentials of growing Samcot 9 cotton variety in Kano State, Nigeria, Science World Journal, 2007:2(1):23-26.

Amidon GE, Houghton ME. The Effect of Moisture on the Mechanical and Powder Flow Properties of Microcrystalline cellulose. Pharm. Res. 1995;12:923-929.

Armstrong NA, "Tableting" In: Aulton ME. (ed), Pharmaceutics: "The science of dosage form design", ELBS, Churchill Livingstone, London, 1990, p.663.

Automashih M, Isah AB, Allagh TS, Ibrahim MA. Heckel and Kawakita analyses of granules of the crude leaves extract of Vergonia Galamensis prepared using polyvinylpyrollidone as binder. Int J Pharm Pharm Sci., 2011;3(4):144-147.

Azubuike CP, Odulaja OJ, Okhamafe OA. Physicotechnical, Spectroscopic and Thermogravimetric Properties of Powdered Cellulose and Microcrystalline cellulose derived from groundnut shells. J Excipients and Food Chem. 2012;3(3):106-115.

Bolhuis GK, Chowhan ZT. Materials for Direct Compaction. In: Alderborn G, Nystron C (eds) Pharmaceutical Powder Compaction Technology, Mercel Dekker Inc., New York, 1996; :419-500.

Bowen FE, Vadino WA. A simple method for differentiating starches. Drug Dev Ind Pharm. 1984;10:505-511.

British Pharmacopoeia, Vol.II, Her Majesty Stationary Office, University Press, Cambridge, London, 2012, A326327, A478-479.

Carlin B, Carter D, Griffiths M, Larner G, Moore K, Rothman $\mathrm{R}$ et al. Joint position paper on pharmaceutical excipient testing and control strategies, 2007, Pharm. Technol. p. 237.
Chuayjuljt S, Su-uthai S, Charuchinda S. Poly(vinyl chloride) filled with microcrystalline cellulose prepared from cotton fabric waste: Properties and biodegradability study. Waste Management and Research, 2010;28:109-117.

Cope RB. "Cotton Seed Toxicity" In: Gupta RC (ed). Veterinary Toxicology: Basic and Clinical Principles, $3^{\text {rd }}$ ed., Academic Press, London, 2018:967-980.

Eichorn SJ, Dufresne Arangueren MA, Marcovich NE, Capadona RJ, Rowan JS, Review: Current International research into cellulose nanofibres and nanocomposites. Journal of Material Science. 2010;45:1-33.

Fox CD, Richman MD, Reier GE, Shangraw RF. Drug Cosm Ind. 1963, 92:161.

France E, Pavlokova S, Sabadkova D, Muselik J. Influence of concentration and type of microcrystalline cellulose on the physical properties of tablets containing Lornelian cherry fruits. Acta Pharma. 2017;67:187-202.

Frenning G, Mahmoodi F, Nordstrom G. An effective medium analysis of confined compression of granular materials. Powder Technology, 2009;194:228-232.

Gomez-Carracedo A, Alvarez-Lorenzo C, Coca R, MartinezPacheco R, Concheiro A, Gomez-Amoza JL. Fractal analysis of SEM images and mercury intrusion porosimetry data for the microstructural characterization of microcrystalline cellulose-based pellets. Acta Mater. 2009;57: 295-303

Gibson LJ. The heirachical structure and mechanics of plant materials. Journal of the Royal Society Interface. 2012;9: 2749-2766.

Gurdag G, Guchi G, Ozgumus S. Grafting of cellulose based materials: A review. J Appl Polym Sci. 2001;80:2267-2272.

Gwon JG, Lee SY, Doh GH, Kim JH. Characterization of chemically modified wood fibers using FTIR spectroscopy for biocomposites. J Appl Polym Sci. 2010;116(6):3212-3219.

Heckel WR. Density-pressure relationship in powder compaction. Transactions of the Metallurgical Society of AIME, 1961;221:671-675.

Kolpak FJ, Blackwell I. (1976). Determination of structure of cellulose II. Macromolecules. 1976:9,273-278.

Kornblum SS, Stoopak SB, A new tablet disintegrating agent; cross linked Polyvinylpyrrolidone. J Pharm Sci. 1973;62(1):43-49.

Kuga S, Brown RM Jr. Silver labeling of the reducing ends of bacterial cellulose. Carbohydrate research. 1988;180:345-350. 
Effect of drying methods on the powder and compaction properties of microcrystalline cellulose derived from Gossypium herbaceum

Natarajan T, Kumaravel A, Palanivelu R. Extraction and Characterization of natural Cellulosic fiber from Passflora foetida stem. Int. J Polym Anal Charact. 2016;21(6):478-485.

Neuman BS. The Flow Properties of Powders, Advances in Pharmaceutical Sciences, Academic Press, London, 1967, p. 181-188.

Nwachukwu N, Ofoefule SI. Effect of drying methods on the powder and compaction Properties of Microcrystalline cellulose obtained from Cocos nucifera. Journal of Pharmaceutical Research International. 2017;20(2):1-15.

Ohwoavworhua FO, Adelakun TA. Some Physical Characteristics of microcrystalline cellulose obtained from raw cotton of Cochlospermum planchonii. Trop. J Pharm Res. 2005;4(2):501-507.

Okhamafe AO, Azubuike PC, Direct Compression of low cost cellulose derived from maize cob. J Pharm Sc Pharmacy Pract. 1994;1:26-29.

Okujagu FT, (ed), Medicinal Plants of Nigeria 'North East Zone, Nigeria Medicine Development Agency, Federal Ministry of Science and Technology, Lisida, Lagos, 2009, p. 66.

Onyem HH, Onyem IM, Usese AI. 'Iron, manganese, cadmium, chromium, zinc and arsenic groundwater contents of Agbor and Owa communities of Nigeria'. Springerplus. 2015,4:104.

Rajkumar R, Mavikandan A, Saravanakumar SS. Physicochemical Properties of alkali-treated new cellulose fiber from cotton shell. Int $\mathrm{J}$ Polym Anal Charact. 2016;21(6):359-364.

Rowe CR, McKillop GA, Bray D. The effect of batch to batch variation on the crystallinity of microcrystalline cellulose. Int J Pharm. 1994;101:169-172.

Setu INM, Mia YM, Lubna JN, Chowbury AA. Preparation of Microcrystalline cellulose from cotton and its Evaluation as Direct Compressible Excipient in the Formulation of Naproxen tablets, Dhaka Univ. J. Pharm. Sci. 2014;13(2):187-192.

Sihtola H, Kyrkxund B, Laamansn L, Palbmius I. Comparison and conversion of viscosity and DP values determined by different methods. Pan. Puu. 1963;45:225-232.
Staniforth JN. "Powder Flow" In: Aulton, ME, Pharmaceutics: The Science of Dosage Form Design, ELBS, Churchill Livingstone, London, 1988, p. 105.

Sun CC. Mechanism of moisture induced Variations in true density and Compaction Properties of Microcrystalline Cellulose. Int J Pharm. 2008;346:93-101.

Suryadi H, Sutriyo, Sari RH, Rosikhoh D. Preparation of microcrystalline cellulose from water hyacinth powder by enzymatic hydrolysis using cellulose of local isolate. J. Young Pharm. 2017;9(1):19-23.

Thoorens G, Krier F, Leclercq B, Evrad B. Microcrystalline cellulose, a direct compression binder in a quality by design environment-A review. Int J Pharmaceutics. 2014;473:64-72.

The United States Pharmacopoeia. The United States Pharmacopoieal Convention, Rockville, USA, 2009, pp. 358, 688-689.

Tomar M, Singh AK, Sinha AR. Powder and Tablet Profile of Microcrystalline cellulose (MCC) of Different Degree of Polymerization. International Journal of Recent Scientific Research. 2016,7(6):12044-12047.

Turki D, Fatah N, Behavior and Fluidization of the Cohesive Powders: Agglomerates Sizes Approach. Brazilian Journal of Chemical Engineering. 2008;25(4):697-711.

Wu YS, Vliet LJ, Frijlink HW, Maarschalk KV. Pore size distribution in tablets measured with a morphological sieve. International Journal of Pharmaceutics. 2007;342:176-183.

Yalli Y, Kostka-Rokosz M, Dvorkin L, Julia Whelan SM. Gossypium Hirsutum/Gossypium Herbaceum, Boston Healing Landscape Project, Boston University School of Medicine. Available at www. Gossypium herbaceum, Wikipedia, Retrieved 20 February 2015.

Yamashiro M, Yausa Y, Kawakita K. An experimental study on the relationship between compressibility, Fluidity and Cohesion of powder solids at small tapping numbers. Powder Technology. 1983; 34:225-231.

Received for publication on $14^{\text {th }}$ February 2018 Accepted for publication on $01^{\text {st }}$ April 2019 